\title{
CERVICAL CANCER - THE PRESENT SCENE
}

O.P. Singh ${ }^{1}$, Gopa Ghosh ${ }^{2}$, Pooja Patil ${ }^{3}$, Veena Khare ${ }^{4}$

\section{HOW TO CITE THIS ARTICLE:}

OP Singh, Gopa Ghosh, Pooja Patil, Veena Khare. "Cervical cancer - the pres ent scene". Journal of Evolution of Medical and Dental Sciences 2013; Vol. 2, Issue 42, October 21; Page: 8099-8109

\begin{abstract}
Recent advances in cervical cancer management with well defined indications of surgery, radiotherapy and chemotherapy have resulted in significant increase in survivors with better QOL. Ongoing recent trials pertaining to further refinement of treatment protocols to make it more cure specific and less morbid will bring more changes in the present scene. This article is a concise review of salient features regarding cervical cancer screening diagnosis \& management at present.
\end{abstract}

KEY WORDS: cancer cervix, screening, diagnosis, and management

INTRODUCTION: Cancer cervix is the second common female [1] malignancy in the world \& leading women cancer in India, which contributes around 1/4th of global incidence. By current trends approximately 500,000 women [1,2] are diagnosed of cancer cervix globally with approximate mortality of 79,000 annually in India. [1] The main reason behind higher incidence in developing countries is lack of awareness about cervical cancer in population, healthcare providers \& policy makers, poor screening program [3] hence late diagnosis \& limited access to cancer centres. Though originally formulated by Zur Hausenbut it is well proved \& well accepted worldwide now that HPV infection [4] is critical for causation of cancer cervix \& HPV DNA detected by hybridization technique [6]in more than95\% cases. Screening with pap smear \& HPVDNA is credited with significant decrease in both incidence \&death rate over past 40 yrs. [4,5] More recent success includes use of combined CT/RT with increased survival in advanced cases. This article is a short summary on cancer cervix screening diagnosis and management.

METHOD: We searched the literature to identify all relevant articles published from 1990 to May 2013 which included Pub Med and Cochrane database) using a combination of the terms "cervical cancer", "diagnosis", and "management". Variables of interest were cervical cancer, surgery, chemotherapy, radiotherapy, chemoradiotherapy, and complications of treatment, follow-up, therapeutic dilemma and challenges. Much of the screening and clinical management discussed in this review was based on meta-analyses, systematic reviews, and phase III randomized controlled trials (RCTs).

\section{REVIEW OF LITERATURE:}

\section{Cervical cancer screening and diagnosis: $[4,5,6,9]$}

The facts, which led to introduction of screening, are:

- It is an important Public health problem in developing countries. [1,2.]

- There is recognized precursor stage that can be treated safely \& effectively.[9]

- Time between appearance of precancerous lesion \&occurrence of cancer cervix is long approx 10 years leaving ample time for detection \& treatment. [4-6]

- Treatment of early lesion is less expen sive compared to invasive cancer.[9] 
- It is estimated that $70 \%$ of cervical cancers are preventable by screening. [4]

- Following methods usually does screening \& diagnosis of cervical cancer:

$>$ Pelvic examination-By PS \&PV.

$>$ Pap test- the examiner prepares smears from scrapings of exocervix, endocervix \& upper vagina. There are two techniques of pap test: [9]

* Conventional- samples are smeared directly on slides

* Liquid based-sample is put in a preservative bottle and smears are stained with tinctorial stains that stain cytoplasmic keratins.

Reporting of pap result is based on Bethseda system2001which as follows: $[5,15]$

1. Normal smear

2. ASCUS (abnormal squamous cells of und etermined significance)

3. LSIL (low grade squamous intraepithelial lesion) includes atypia, CIN1

4. HSIL (high grade squamous intraepithelial lesion), includes CINII, III, CIS.

5. Invasive squamous cell cancer.

$>$ HPV DNA test on a cervical smear[6,Cervical cancer screening Wikipedia]

$>$ Colposcopy -done by a colposcope, which gives magnified view of cervix \& vagina.

$>$ Colposcope enables two types of examination:[7,36]

* Colposcope assisted visual examination $\rightarrow$ VILI(visual inspection with Lugols iodine) $\rightarrow$ VIAA (visual inspection with acetic acid)

* Colposcope directed punch biopsy usually four quadrant from exocervix [15]

$>$ ECC-Endocervical curettage done mainly if Pap test indicates a glandular lesion \&after therapeutic conisation of exocervix. [7]

$>$ LEEP-uses electric current passed through a thin wire hook removes tissue for examination. A LEPP may be used to treat precancer or early stage cancer. [8]

$>$ Conisation-removes cone shaped area of abnormal tissue from cervix, also treat precancer or early stage cancer. [15]

> Imaging studies like X-ray chest, CT scan, MRI, PET allows evaluation of extent of disease. $[12,36]$.

$>$ PET-CT SCAN-This is one of the latest imaging tool which is an integration/fusion of functional (PET) and anatomic imaging (CT Scan) with greater sensitivity and specificity than conventional anatomic and morphologic imaging , has fast emerged as an invaluable tool in staging/ restaging particularly in early stages, assessing response to therapy, early detection of recurrence in asymptomatic patients and deciding or modifying treatment plan in cancer cervix since its commercial inception in early 2000's. [43,44]

$>$ Cystoscopy \&Proctoscopy when indicated. [10]

Progress of cervical cancer from CIN 1(LSIL) to invasive squamous cell cancer is as shown in the flow chart Fig1: [5] 


\section{REVIEW ARTICLE}

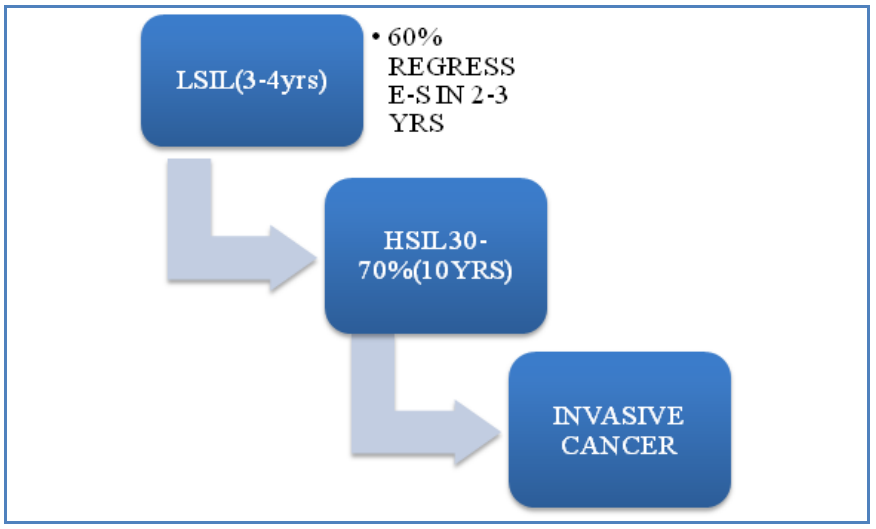

Fig. 1

MANAGEMENT: Management of cervical cancer is based on evaluating and treating primary disease and potential areas of spread i.e. parametrium pelvic, paraortic nodes and distant metastatic sites. $[7,8,10,15,27]$

\begin{tabular}{|c|c|c|}
\hline Stage & Pelvic LN (\%) & Para-aortic \\
\hline Ia1 & 0.5 & 0 \\
\hline Ia2 & 4.8 & $<1$ \\
\hline I b & 15.9 & 2.2 \\
\hline II a & 24.5 & 11 \\
\hline Ilb & 31.4 & 19 \\
\hline III & 44.8 & 30 \\
\hline IVa & 55 & 40 \\
\hline
\end{tabular}

TABLE-1: Stage wise Lymph node metastasis rates: [15]

Surgery or Radiotherapy (RT) broadly treats cervical cancer with equivocal results in early stages (I-IIA) [11, 12, 15, 18] \& chemoradiotherapy in late stages (IIB-IVA). [19, 21]. Few prospective randomized studies had been carried out to compare Surgery/RT in early disease. Equivalent outcome $80-90 \%$ 5year survival reported with either $[11,13,32]$, but in most studies Surgery arm patients (stage IB-IIA) required postoperative RT [15-19,28-31] Therapeutic modalities used in various stages are: $[7,8,10,15,27]$

* Surgery in early stages (I-IIA)

* Radiotherapy in all stages - In early stages when there is medical contraindication to surgery, extensive multifocal in situ disease, bulky IB-IIA disease with higher rates of recurrence

* Chemoradiotherapy in late stages (IIB-IVA)

* Chemotherapy (CT) in metastatic disease. (IV) 


\section{TABLE 2 - Carcinoma in Situ (stage 0)}

- Loop electrosurgical excision procedure (LEEP)

- Laser surgery

- Conization

- Cryosurgery

- Total hysterectomy for women who cannot or no longer want to have children.

- Internal radiation therapy / brachytherapy for women who cannot have surgery.

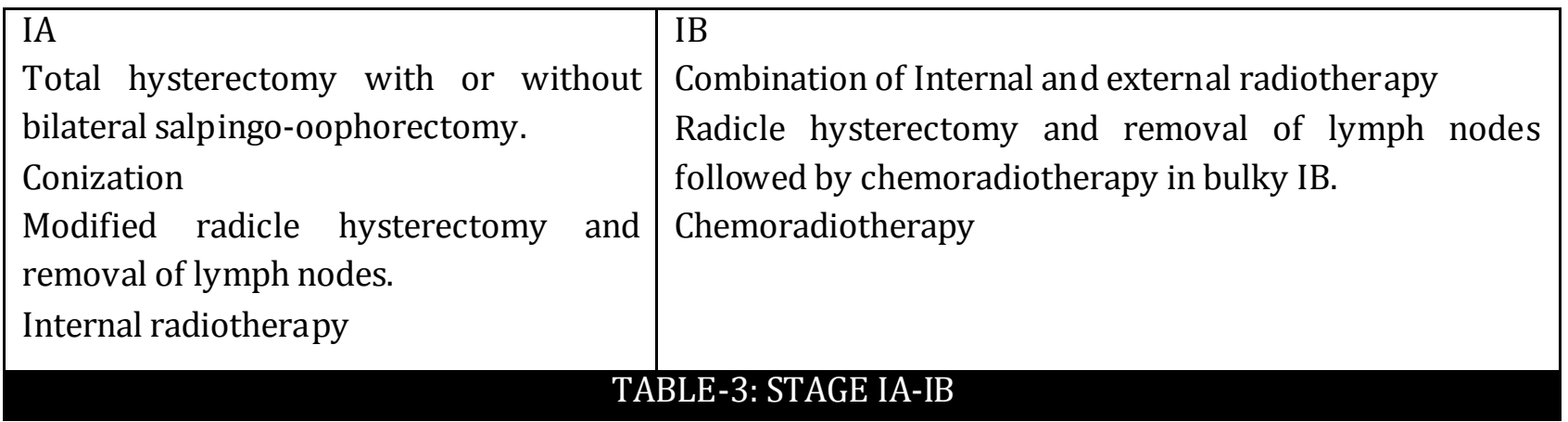

\section{TABLE-4: Stage-IIA}

- A combination of Internal radiation therapy and external radiation therapy plus chemotherapy

- Radicle hysterectomy and removal of lymph nodes.

- Radicle hysterectomy and removal of lymph nodes followed by chemoradiotherapy.

\begin{tabular}{|l|l|}
\hline $\begin{array}{l}\text { IIB-IVA } \begin{array}{l}\text { External and internal radiotherapy } \\
\text { Combined with chemotherapy. }\end{array} \\
\text { IVB } \\
\text { Radiotherapy as palliative therapy to relieve } \\
\text { Symptoms caused by the cancer and improve } \\
\text { quality of life. } \\
\text { Chemotherapy }\end{array}$ \\
\hline \\
TABLE-5: STAGE IIB-IVA
\end{tabular}

Some Surgical procedures in Cancer Cervix:

$>$ Fertility sparing: (stage 0-stage IAI) [7,34]

- Superficial ablative surgeri es like(Cryo, laser, LEEP)

- Conization

- Trachylectomy

> Total Hysterectomy \& Modified radicle hysterectomy (stage0-IA2)[7,8]

$>$ Radicle Hysterectomy or Wertheim's Hysterectomy-(stage IB-IIA) [32]

$>$ Laparoscopic/robot assisted radicle Hysterectomy [34]

$>$ Pelvic Exenteration-Extensive recurrent disease. [27]

$>$ Pelvic lymphadenectomy [33]

$>$ Oophoropexy- for preservation of ovarian function in patients undergoing radicle hysterectomy or RT. [15] 
Safety and efficacy of modern minimally invasive surgical techniques like laparoscopic or robotic assisted radicle trachylectomy and radicle hysterectomy for early stage cervical cancer with the objective of reducing perioperative morbidity, hospital stay, blood loss and improving cosmesis have gradually evolved and rapidly gaining popularity in surgical management of this disease. [39, 40]

\section{INDICATIONS OF SURGERY IN CANCER CERVIX: [27-34]}

1. EARLY STAGE \& EARLY AGE Surgery alone is curative for stage 0 to stage IAI, for patients who desires to preserve fertility, fertility sparing surgeries can be done in these stages, surgery alone when curative may be preferred treatment in young age as by maintaining the vaginal anatomy and function preserves normal sexual function.

2. Early stage (IA2-IIA, non bulky) -Surgery alone or Surgery followed by Radiotherapy when indicated

3. Early stage (IB2 -IIA bulky) - Chemoradiotherapy followed by surgery when chemoradiotherapy alone is not sufficient to cure the disease

4. Recurrent disease after definitive treatment.

5. Pelvic lymphadenectomy/pelvic lymph node sampling- done by some -in early stages when simple hysterectomy is planned to assess lymph node involvement. [33]

\section{RADIOTHERAPY (RT) IN CERVICAL CANCER:}

Indications: $[8,15,18,16,20,21,22,25$.

- Definitive Radiotherapy in all stages, early stages with pelvic nodal $[8,15]$

Involvement, poorly differentiated tumour, deep stromal invasion in bulky disease/barrel shaped cervix, medical contraindications to surgery definitive radiotherapy is preferred treatment compared to surgery alone in order to reduce probability of recurrence.

- Post operative

- Pre operative (In bulky early disease.)

- RT for recurrence

- Palliative RT

Indications of post operative EBRT in early disease: [17, 18, 19,]

○ Positive pelvic lymph nodes

○ Positive surgical margin

○ LVSI

○ Deep stromal invasion (Bulky tumour)

- Positive parametrium

- Doubt regarding adequacy of surgery/HP

\section{TYPES OF RT:}

1. External beam RT (EBRT) $[15,20,26]$

Equipments:

Telecobalt and Linear accelerator 
EBRT (External beam radiotherapy) DOSE:

45-60 Gy to whole Pelvis [15]

Total Dose- EBRT+ICRT (INTRACAVITARY RT) $\rightarrow$ 75-80Gy (LDR/low dose rate equivalent) to point A.

2. Brachytherapy (BT) / Internal. [7,8,10,20,21,22]

- Sealed Radioactive sources are placed close to or in contact with target tissue.

- Sources like, 226Ra, 60 CO, 137Cs, 192Ir are used which shows rapid fall off dose beyond target.

- 2 types of brachytherapy application are done in cervical cancer:

A. Intracavitary (ICRT) (Placement of radioactive sources inside predefined applicators in uterine cavity and vagina with special geometry)

B. Interstitial (placement of radioactive sources in needles inserted through perineal template

Advantages of INTRACAVITARY RT (ICRT) [19, 23, 24]

1. High local dose, with higher local control rate.

2. Better sparing of normal tissues

3. Preserves normal anatomy.

4. Responsible for most of the cure.

Present day Intracavitary radiotherapy is based

On Manchester system which: [15, 20, 21, 23, 27]

- Defines two points A \&B

- Designed a .set of applicators and their loading which would give a constant dose rate irrespective of combination of applicator \& loading used.

- Formulated a set of rules regarding activity and positioning of radium sources in the tandem \& vaginal ovoids for desired dose rate.

- Total dose to point A 75-80 Gy LDR equivalent.

- Pelvic side wall dose 45-60 Gy

- Dose rate $-57.5 \mathrm{R} / \mathrm{hr}$

- Bladder dose<80 Gy

- Rectal dose $<75$ Gy

Equipment: High dose rate(HDR) remotely after loading brachytherapy machine, which delivers, desired dose in 3-6 Weekly fractions of few minutes at the dose rate of $120-180 \mathrm{GY} / \mathrm{hr}$ or 23Gy/minute are most widely used these days.[15,20,22,25.]

DOSE BRACHYTHERAPY (BT): [12, 15, 20, 22]

1. STAGE IA (microinvasive) - Treated with BT alone and the usual dose HDR is35-42 Gy in 5-6 insertions of $7 \mathrm{GY} /$ wkly fractions.

2. EARLY STAGES (IB-IIA) - BT, EBRT (6-7 fractions of 7-6GY/fr).

3. LATE STAGES-EBRT, BT (4 fractions of 6-7GY/fr). Interstitial brachytherapy Indications $[10,15,28]$ 
$>$ IB, IIB disease $\&$ above in conditions mentioned below:

- Bulky parametrial disease as boost

- Post operative vault recurrence

- Persistent or recurrent disease post EBRT +ICA

- ICA (Intracavitary applications) not possible in conditions:

$>$ Distorted anatomy

$>$ Os not identifiable

$>$ Narrow vagina with obliterated fornices.

Besides the above mentioned time tested and well accepted conventional Radiotherapy techniques, Pros \& cons of latest EBRT techniques like 3D-CRT and IMRT are under extensive evaluation with respect to increasing therapeutic ratio(escalating dose to target and minimizing doses to normal tissue) as boost technique mainly for residual parametrial and para aortic disease and as potential substitute of brachytherapy in select cases where brachytherapy is not feasible like post radicle hysterectomy. [41] Accuracy of these techniques though questioned by limitations like precision of contouring, reproducibility, organ motion, under dosing etc. $[12,41]$ at present but in days to come, dose painting (2D) and dose sculpting (3D) by IMRT based on biology based imaging (PET-CT, SPECT, MR Spectroscopy) and concept of BTV (Biological target volume) in addition to GTV, CTV and PTV are expected take IMRT to a new height in the new millennium. [42]

\section{CHEMOTHERAPY (CT) IN CANCERCERVIX/Indications: [7, 8, 25, 26]}

* Used mostly concurrently (as Radiosensitiser)with RT for bulky IB,IIA disease when treated with RT alone.[14,18]

* Postoperative chemoradiotherapy in early disease with high-risk features for recurrence as mentioned earlier. $[27,30]$

* Concurrently for advanced IIB-IVA disease. [19,21]

* Palliative CT in metastatic disease $[8,15]$

* As NACT (Neoadjuvant) for IB-IIIB to downstage the disease \& improve outcome of Surgery /RT but is still in trial phase. [23,24]

* Platinum compounds or Taxanes used singly or in various combinations are the well accepted and most commonly used chemotherapeutic drugs at present. [47]

* Cisplatin or Cisplatin +5FU in concurrent setting, Paclitaxel + Cisplatin, Docetaxel +Carboplatin, Cisplatin + Gemcitabine are some common regimens currently in practice as NACT. $[46,48,49]$

DISCUSSION: Early screening can prevent cancer cervix by diagnosing $100 \%$ curable premalignant stages and early stages, and adequate treatment of early stage disease based on well-defined management guidelines for integrating Surgery, radiotherapy and chemotherapy can result in very high survival rates, as reflected in the Table 6: [3-6, 8, 10, 15, 18, 27, 32]

\begin{tabular}{|c|c|}
\hline STAGE & 5 YR SURVIVAL \\
\hline 0 & $93 \%$ \\
\hline IA & $93 \%$ \\
\hline
\end{tabular}




\begin{tabular}{|c|c|}
\hline IB & $80 \%$ \\
\hline IIA & $63 \%$ \\
\hline IIB & $58 \%$ \\
\hline IIIA & $32 \%$ \\
\hline IIIB & $18 \%$ \\
\hline IVA & $16 \%$ \\
\hline IVB & $15 \%$ \\
\hline \multicolumn{2}{|c|}{ TABLE -6: STAGE WISE SURVIVAL RATES [26] } \\
\hline
\end{tabular}

\section{Some therapeutic dilemma involving cervical cancers is:}

$>$ Choosing \& designing individualised Treatment protocol out of existing stage wise treatment options. [36, 37].

$>$ Management is multidisciplinary hence may result in to selection bias \& poor interdisciplinary referral. [38]

$>$ Offering cost effective treatment $\rightarrow$ Use of high-end technology both in diagnosis \& therapy should be need based. [36]

\section{SOME CHALLENGES:}

$>$ Further refinement of existing guidelines and formulating more cure specific guidelines. [38]

$>$ Increasing specificity of Pap smear (liquid based pap, Immunohistochemistry on pap). [9,35]

$>$ Enhancing mass awareness regarding very high cure \& survival rate by early diagnosis \& treatment. $[4,5]$

$>$ Implementation of screening at primary level [3]

CONCLUSION: Better insight in to etiology, pathogenesis and technological breakthrough in Surgery, Radiotherapy \& chemotherapy have culminated in to very high cure \& survival rate of early cervical cancer with good quality of life.[7,8,9,10,11,19,2627,28,32]Ongoing trials to make treatment more cure specific will increase cure rates of this most dominant female cancer in developing countries further.

\section{REFERENCES:}

1. Cancer cervix incidence-www. metindia. net/health information.

2. Anand Kumar. The magnitude of cancer cervix in India. Indian j of Med Res. 130: Sept 2009; p219-221. [PUBMED].

3. DCCP-A model demonstration program for implementation of cervical cancer screening guidelines. NCCP-WHO: 2006; www. slideshare. net /cervical-cancer-screening-15771193.

4. Basu P \& Chowdhury D. Cervix cancer screening \&HPV vaccination: a comprehensive approach to cervical cancer control. Indian J of Med Research130: sept 2009, 241-6. [PUBMED].

5. Bishop A, Sherri's tsu. Cervical dysplasia treatment: key issues for developing countries Bulletin of Pan America health organization. Dec1996: 30(4); (378-86).

6. Cruickshank ME, Cubie HA. Cervical screening: primary human papillomavirus testing. BJOG 2012; 119:650-657. 
7. Www. cancer. gov/ treatment/ cervical. Cervical cancer treatment: NCI.

8. Treatment options of cancer cervix by stage, www. cancer. org April 2013.

9. En. wikipedia. org/wiki/Pap test.

10. Scottish Intercollegiate guidelines network. Management of cervical cancer: A national clinical guideline.

11. F. Landoni, A Marco. Radicle Surgery vs RT for stage IB-IIA Ca cervix. Lancet. 1997; 350:53540.

12. Akila vishwanathan, Advances in cancer cervix management: (ASTRO). www. astro. Org/uploaded files/vishwanathan/cervical. Pdf.

13. Rad Hysterectomy vs RT for stage IB Sq cell cancer, Cancer, vol, 68, Issue2, 272-277

14. William Apeters III, P. Y. Liu. Concurrent CT/RT compared to Pelvic R Talone in high-risk early stage cancer cervix. J. Of clinical oncol. Apr 1 2000: vol18; 1606-1613[PUBMED]

15. Carloz A. perez, Brian D. Kavanagh. Principles \& Practice of Radiation Oncol: $4^{\text {th }} \& 5^{\text {th }}$ ed. ; 1546-1596:Lippincot Williams \& wilkins.

16. Smith Kristy, Breuhl et al. Post op RT for cancer cervix after simple hyst for benign conditions. Am. J of clinical Oncol. June2010:vol 33;3229-232[PUBMED]

17. Chen Sw, Lidng et al. PORT in inv. ca cervix foll simple hyst. Jpn. J. of clin oncol. Sep 2003:33(9); 477-81. [PUBMEDCENTRAL]

18. Thomas. E. Bucker et al. The treatment of early cervical cancer. Gynecologic oncol. 2007:104; 665-669.

19. J. Green, J Kirwan. Concomitant CT/RT in cervical ca. The Cochrane lib: 2012; issue (2). [Cochrane]

20. Daniel G. Petereit, Robert Pearcey. Literature analysis of HDR brachyfractionation schedules in treatment of cervical cancer. International J. of Radiation Oncol Biol Physics. Jan1999, 43(2); p 359-366.

21. T. T. Singh, I. Y. Singh, Role of chemoradiation in advanced cervix ca. India Journal of Cancer. Sept 2003:40(3); 101-7[PUBMED]

22. Patel FD, Sharma S. C. et al LDR vs HDR in ca cervix-International J of Radiat oncol, Biol, Phys 1993, 28; 335-341.

23. Sardi Juan et al Neo adjuvant CT in cervical ca a new trend. Current opinion in O\&G. Feb 2003, 17(1); 43-47.

24. JV Kumar, DC Donval et al Locally advanced cancer cervix treated with NACT followed with Surgery; J. of gynecologic oncology; 2009

25. Cervical cancer- RT. www. iscort. org.

26. Survival rates of Cancer Cervix by stage- 7 th edition AJCC staging manual

27. Cervix Cancer, NCI /Treatment options review May15, 2013

28. Cervical cancer/Pg4cancer network, www. cancernetwork. com/ cancer management.

29. Andrew Pscisson, John. t. Soper et al Adjuvant RT following radicle hysterectomy for patients with stage IB, IIA cervical cancer. Gynecologic Oncology. June 1990: Vol 37, (3); 390-395.

30. liu MT, HSU. JC. Prognostic factors affecting outcome of early cervical cancer treated with radicle hysterectomy \&postop adj. RT. Eur J Cancer. 2008 mar: 17(2); 174-81.

31. A. yessaian, A. magistris. Rad. Hysterectomy followed by Postop therapy in stage IB2 cervical cancer. Gynaec. Oncol. Jul 2004:94(1); 61-66 
32. KJ Harrington, H. Thomas. Rad Sur vs RT for IB-IIA cervical cancer. Lancet. Oct1997: vol 350(9085); 1179

33. JM Childers, K. Hatch. Laparoscopic pelvic lymphadenectomy in management of cancer cervix. Gynec oncol. VolOct 1992:47(1); 38-43

34. Surgeries for cervical cancer \& precancer-www. cancer. org

35. En. Wikipedia. Org/wiki/cervical cancer.

36. ACOG: Practice BulletinsNo. 35, Diagnosis and treatment of Cervical Cancer May2002vol. 99 (5), Part1; p855-86

37. G. Pentheroudakis, R. Orecchia. Cancer, fertility \&Pregnancy, ESMO Clinical Practice guidelines. Annals Onco, vol21 (5) p266-273.

38. www. e guidelines. co. uk, /suspected cancer-Referral guid elines for suspected cancer

39. Sobiczewski P, Bidzinskim, Derlatka P. Early cervical cancer managed by laparoscopy and conventional Surgery: comparison of treatment results Int. J of Gyneco Cancer. Nov 2009: $19(8) ; 1390-5$

40. Mazzoni. M, Spina, Pernio la G. Laparoscopic Sur in treatment of stage IIB cervical cancer after NACT: A Case report \& review of lit. Eur J. gynaecol oncol. 2003:24(5) 393-7.

41. Loiselle C, Koh WJ The emerging use of IMRT Ca Cervix treatment. www. ncbi. nim. nih. gov/Pubmed2010/21147905.

42. C. Clifton ling, John Humm, Steven Larson. Towards multidimensional RT(MD-CRT): Biological imaging \& conformality Int J of Radiation Oncol, Biol, Phy: 2000:47(3); 551-560.

43. Jover R, Lourido D. Role of PET=CT in Cervical Cancer. Gynecol oncol. Sep 2008:110(3 suppl2); S55-9.

44. David W. Townsend Combined PET-CT: The historical aspect. Seminars I US, CT, MR :2008

45. D. Termoungruanglert, D. Tresukosol, AVasuratna. Neoadjuvant Gemcite \& Cisplatin followed by radicle Surgery in bulky sq cell ca of stage IIB Gynecol oncol. 2005May: 97(2);576-81

46. Angelo Vacca, Domenico Ribatti, Monica Iurlaro, Francesca Merchionne, Beatrice Nico, Docetaxel Versus Paclitaxel for Anti-angiogenesis Journal of Hematotherapy \& Stem Cell Research. February 2002, 11(1): 103-118.

47. D. T. Rein, C. M. Kurbacher, M. Breidenbach, T. Schöndorf, T. Schmidt, E. König. Weekly Carboplatin and Docetaxel for Locally Advanced Primary and Recurrent Cervical Cancer: A Phase I Study. Gynecologic Oncology. Volume 87, Issue 1, October 2002, Pages 98-103.

48. Nagao S, Fujiwara K, Oda T, Ishikawa H, Koike H, Tanaka H, Kohno I. Combination chemotherapy of docetaxel and carboplatin in advanced or recurrent cervix cancer. A pilot study. Gyneccol Oncol. 2005:96; 805. 


\section{REVIEW ARTICLE}

\section{AUTHORS:}

1. O.P. Singh

2. Gopa Ghosh

3. Pooja Patil

4. Veena Khare

\section{PARTICULARS OF CONTRIBUTORS:}

1. Professor, Department of Radiotherapy \& Clinical Oncology, Gandhi Medical College, Bhopal, India.

2. Consultant, Department of Radiotherapy \& Oncology, J.K. Hospital \& L.N. Medical College \& RC, Bhopal, India.

3. Assistant Professor, Department of Obstetrics \& Gynecology, J.K. Hospital \& L.N. Medical College \& RC, Bhopal, India.
4. Assistant Professor, Department of Obstetrics \& Gynecology, J.K. Hospital \& L.N. Medical College \& RC, Bhopal, India.

\section{NAME ADDRESS EMAIL ID OF THE CORRESPONDING AUTHOR:}

Dr. Gopa Ghosh, B-16, Pragati Complex, Jawahar Chowk, City Depot Square, Bhopal - 462003, M.P.

Email - gopaghosh571@yahoo.in

Date of Submission: 28/09/2013.

Date of Peer Review: 29/09/2013.

Date of Acceptance: 11/10/2013.

Date of Publishing: 17/10/2013 